\title{
PREPARAÇÃO E CARACTERIZAÇÃO DE COBALTITA DE FERRO PARA DEGRADAÇÃO DE POLUENTE EM FOTO-FENTON SOLAR
}

\section{Letícia Camargo Munhoz; William Leonardo da Silva²; Jivago Schumacher de Oliveira $^{3}$}

\section{RESUMO}

Este projeto tem como objetivo principal produzir o espinélio cobaltita de ferro $\left(\mathrm{FeCo}_{2} \mathrm{O}_{4}\right)$ para uso em reações heterogêneas de foto-Fenton sob irradiação visível e solar, visando a decomposição de poluentes orgânicos recalcitrantes em soluções aquosas. A Cobaltita de ferro foi sintetizada a partir de uma rota solvotérmica, utilizando cloreto de cobalto $\left(\mathrm{CoCl}_{2} .6 \mathrm{H}_{2} \mathrm{O}\right)$ e cloreto férrico $\left(\mathrm{FeCl}_{3} .6 \mathrm{H}_{2} \mathrm{O}\right)$ como percursores. $O$ material formado foi caracterizado até o momento por difração de raios-X (DRX) e espectroscopia de infravermelho com transformada de Fourier (FTIR). Para os ensaios catalíticos será utilizado o corante azo Amaranto. As reações foto-Fenton serão irradiadas sob luz artificial visível e solar. Os resultados de DRX e FTIR se apresentam em concordância, confirmando a formação da fase única do cristal $\mathrm{FeCO}_{2} \mathrm{O}_{4}$ com estrutura espinélica. Espera-se que o material desempenhe elevada eficiência na decomposição do poluente orgânico, possibilitando uma inovação na área de síntese de materiais para aplicação ambiental.

Palavras-chave: Tratamento de Efluentes; Foto-Fenton Heterogêneo; Catalisador; Corante.

Eixo Temático: Sociedade e Ambiente (SA)

\section{INTRODUÇÃO}

Atualmente com a substancial incorporação dos Objetivos de Desenvolvimento Sustentável (ODS) e a consciência ambiental, surge a necessidade de ações que reduzam o impacto negativo da atividade antropogênica sobre a natureza. Grande parte dos problemas ambientais advém de processos industriais agressivos ao meio ambiente, em que há uma elevada geração de efluentes líquidos e emissões gasosas, além de resíduos sólidos, que promovem grande impacto à natureza.

\footnotetext{
${ }^{1}$ Bolsista Iniciação Científica - Universidade Franciscana (UFN), camargo.leticia@ufn.edu.br

2 Professor do Curso de Engenharia Química e do Programa de Pós Graduação em Nanociências -

UFN,w.silva@ufn.edu,br

${ }^{3}$ Professor do Curso de Engenharia Ambiental e Sanitária - UFN, jivago@ufn.edu.br
} 
Os processos industriais da produção de fármacos, pesticidas, tintas, têxteis, destilarias, fermentação para produção de etanol, aminoácidos e leveduras são exemplos de processos que produzem alta vazão de efluentes líquidos contendo elevada carga de contaminantes orgânicos, cor acentuada e diversos produtos tóxicos ao homem e ao meio ambiente. O tratamento desses efluentes pelos processos tradicionais de lodos ativados ou qualquer combinação de processos biológicos, físicos e químicos, não são sufucientes ou adequados para atingir os padrões exigidos com resultados satisfatórios.

Nesse sentido, é notório os esforços recentes de pesquisadores por métodos de tratamento mais eficientes. Os Processos Oxidativos Avançados (POAs) têm sido amplamente testados e aplicados na degradação de poluentes orgânicos em soluções aquosas geradas pelos setores industriais (OLIVEIRA et al., 2018). Os Processos Oxidativos Avançados baseiam-se na geração in situ de poderosos agentes oxidantes, como os radicais hidroxila $(\bullet \mathrm{OH})$. Estes processos normalmente ocorrem em condições ambientes, tanto de temperatura quanto de pressão e podem promover oxidação completa de poluentes orgânicos com a formação de moléculas de $\mathrm{CO}_{2}, \mathrm{H}_{2} \mathrm{O}$ e alguns sais inorgânicos ao final do processo ( DA SILVA et al., 2013; BOKARE e CHOI, 2014; BOCZKAJ e FERNANDES, 2017).

Entre os POAs o sistema foto-Fenton vêm sendo vastamente estudado em sistemas de reações heterogêneas, por apresentarem melhores condições de operação e superar deficiências apresentadas nos sistemas homogêneos. Este sistema recebe grande atenção por seu alto rendimento de radical hidroxila gerado pela combinação de peróxidos (principalmente o peróxido de hidrogênio), íons ferrosos e irradiação em meio ácido (ZHOU et al., 2014; MAEZONO et al., 2011; OLIVEIRA et al., 2019). Possui etapas e reagentes de natureza segura e ambientalmente amigável, princípios de funcionamento relativamente simples e tempo curto de reação, sendo o mais usado em aplicações ambientais. (VELÁSQUEZ et al., 2014; OLIVEIRA et al., 2019; OLIVEIRA et al., 2016).

Com isso, existe uma intensa busca por novos catalisadores, com melhores propriedades catalíticas para as aplicações em reações de decomposição de poluentes orgânicos em sistemas foto-Fenton heterogêneo. Trabalhos com aplicação do espinélio cobaltita de ferro $\mathrm{FeCO}_{2} \mathrm{O}_{4}$ em processos fotocatalíticos de foto-Fenton solar visando à melhoria da degradação de poluentes orgânicos a partir de solução aquosa, ainda não foram relatados na literatura. Dessa forma, este projeto tem como objetivo principal produzir o espinélio cobaltita de ferro $\mathrm{FeCO}_{2} \mathrm{O}_{4}$ para uso em reações heterogêneas de foto-Fenton sob irradiação visível e solar, visando a decomposição de poluentes orgânicos recalcitrantes em soluções aquosas.

\section{METODOLOGIA}

\subsection{Preparação do Catalisador}

Cobaltita de ferro $\left(\mathrm{FeCO}_{2} \mathrm{O}_{4}\right)$ foi sintetizada a partir de uma rota solvotérmica, utilizando cloreto de cobalto $\left(\mathrm{CoCl}_{2} \cdot 6 \mathrm{H}_{2} \mathrm{O}\right)$ e cloreto férrico $\left(\mathrm{FeCl}_{3} \cdot 6 \mathrm{H}_{2} \mathrm{O}\right)$ como percursores. Etilenoglicol $\left(\mathrm{C}_{2} \mathrm{H}_{4} \mathrm{O}_{2}\right)$ foi utilizado como solvente. A razão 
estequiométrica dos sais usada foi de 1:2= Fe:Co. Assim, $8 \mathrm{mmol}$ de cloreto de cobalto e $4 \mathrm{mmol}$ de cloreto férrico foram dissolvidos em $120 \mathrm{~mL}$ de etilenoglicol sob agitação, seguido da adição de $60 \mathrm{mmol}$ de acetato de sódio $\left(\mathrm{NaC}_{2} \mathrm{H}_{3} \mathrm{O}_{2} \cdot 3 \mathrm{H}_{2} \mathrm{O}\right)$. A solução foi transferida para o interior de copos de teflon acoplados a autoclaves de aço inoxidável, e em seguida submetidas a uma temperatura de $200^{\circ} \mathrm{C}$ por um período de 10 horas. Após, o material obtido foi lavado e seco a $80^{\circ} \mathrm{C}$.

\subsection{Caracterização do catalisador}

O material formado foi caracterizado por difração de raios-X (DRX), usando um difratômetro Rigaku Miniflex 300, com radiação Cu-Ka $(\lambda=1,5418 \AA)$, fonte de energia com $30 \mathrm{kV}$ e $10 \mathrm{~mA}$, com passo de $0,03^{\circ}$ (em 20) e tempo de aquisição de 0,9 s. O espectro de FTIR da amostra comprimida em pelete de $\mathrm{KBr}$ (10 mg CoFe2O4 / 300 mg KBr) foi registrado por um espectrômetro Shimadzu IR-Prestige21. Pretende-se ainda realizar as caracterizações de área específica, volume de poros e tamanho médio dos poros a partir da técnica de adsorção/dessorção de nitrogênio com o aparelho Micromeritics ASAP 2020, onde a amostra será previamente tratada a $300^{\circ} \mathrm{C}$ sob vácuo. E também obter as imagens das partículas da amostra por microscopia eletrônica de varredura (MEV, FEI Inspect S50).

\subsection{Ensaios catalíticos}

Como molécula modelo poluente, será utilizado o corante azo Amaranto (CAS n. 915-67-3, $\mathrm{C}_{20} \mathrm{H}_{11} \mathrm{~N}_{2} \mathrm{Na}_{3} \mathrm{O}_{10} \mathrm{~S}_{3}$, massa molecular: 604,47 g mol-1) As reações fotoFenton serão realizadas usando um recipiente de vidro $(250 \mathrm{~mL})$ disposto sobre um agitador magnético, e irradiadas sob luz artificial visível e solar. Para os ensaios sob luz visível, será usado uma lâmpada fluorescente comercial (85 W, Empalux, intensidade de irradiação: $3,8 \mathrm{~mW} / \mathrm{cm}^{2}$ ). Para os experimentos sob luz solar, a solução será exposta ao ar livre em um dia sem nuvens e os dados de irradiação deste dia, durante 0 horário utilizado para 0 ensaio será considerado. Os experimentos serão realizados empregando uma relação de 0.5 gramas de catalisador por litro de solução de corante. A concentração do corante na solução será de $75 \mathrm{mg} \mathrm{L}^{-1}$. A solução será ajustada ao $\mathrm{pH}=3 \mathrm{com}$ ácido sulfúrico diluído em água destilada $\left(0,1 \mathrm{~mol} \mathrm{~L}^{-1}\right)$. Antes de iniciar a reação foto-Fenton, a solução (100 $\mathrm{mL}$ ) será agitada na presença do catalisador até atingir o equilíbrio de adsorção. Então, $8 \mathrm{mmol} \mathrm{L} \mathrm{L}^{-1}$ de peróxido de hidrogênio $\left(\mathrm{H}_{2} \mathrm{O}_{2}\right)$ será adicionado à solução, e esta exposta a irradiação (visível ou solar), dando início à reação foto-Fenton. As amostras serão retiradas no decorrer da reação com auxílio de uma seringa e centrifugadas para separação do catalisador da solução. A descoloração da solução será determinada pela leitura da cor em um espectrofotômetro UV-vis (Bel Photonics, SP1105), no comprimento de onda de máxima absorbância, $525 \mathrm{~nm}$. A cinética de descoloração da solução será expressa pela razão $C / C_{0}\left(=A / A_{0}\right)$ em função do tempo, onde: $A_{0}$ e A são as absorbâncias da solução de corante inicial e no tempo de reação $t$, respectivamente. Os ensaios serão realizados em triplicata. 


\section{QUFN}

\section{RESULTADOS E DISCUSSÕES}

\section{Caracterização do catalisador}

A Figura 1 apresenta o difratograma de raios-X (DRX) do material produzido $\left(\mathrm{FeCO}_{2} \mathrm{O}_{4}\right)$. Foi possível observar que houve a formação da fase única $\mathrm{FeCO}_{2} \mathrm{O}_{4}$, com picos de difração localizados em $2 \theta$ a $18.29^{\circ}, 30.08^{\circ}, 35.55^{\circ}, 43.06^{\circ}, 47.03^{\circ}$, $56.97^{\circ}$ e $63.22^{\circ}$ de acordo com o cartão JCPDS número 22-1086 (LIU et al., 2017). Além disso, não são observados picos de impurezas no difratograma, evidenciando a formação de um material puro de fase única da $\mathrm{FeCo}_{2} \mathrm{O}_{4}$.

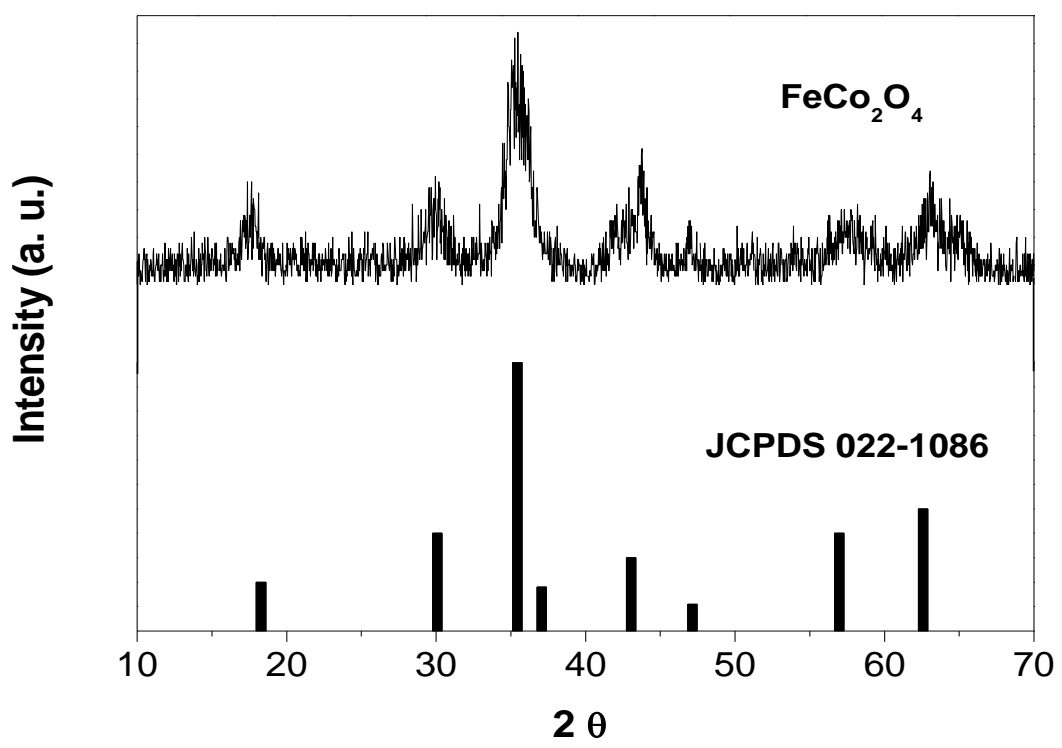

Figura 1 - Difratograma de raios- $\mathrm{X}(\mathrm{DRX})$ da $\mathrm{FeCO}_{2} \mathrm{O}_{4}$.

O espectro de FTIR da $\mathrm{FeCO}_{2} \mathrm{O}_{4}$ está apresentado na Figura 2. A banda larga em cerca de $3423 \mathrm{~cm}^{-1}$ foi atribuída ao modo de alongamento do grupo O-H da água livre e absorvida. Bandas em $1180 \mathrm{~cm}^{-1} \mathrm{e} 1700 \mathrm{~cm}^{-1}$ estão associados à presença de umidade absorvida na amostra de $\mathrm{FeCo}_{2} \mathrm{O}_{4}$. Uma forte absorção próxima a $575 \mathrm{~cm}^{-1}$ é atribuída ao modo de vibração do complexo de grupos de cátions octaédricos $\left(\mathrm{Co}^{2+}-\mathrm{O}^{2-}\right)$ de partículas de cobaltita de ferro (ZHAO et al., 2021).

$O$ resultado de DRX se encontra em concordância com os dados de FTIR, que confirmaram a formação do cristal $\mathrm{FeCO}_{2} \mathrm{O}_{4}$ com estrutura espinélica. 


\section{QUFN}

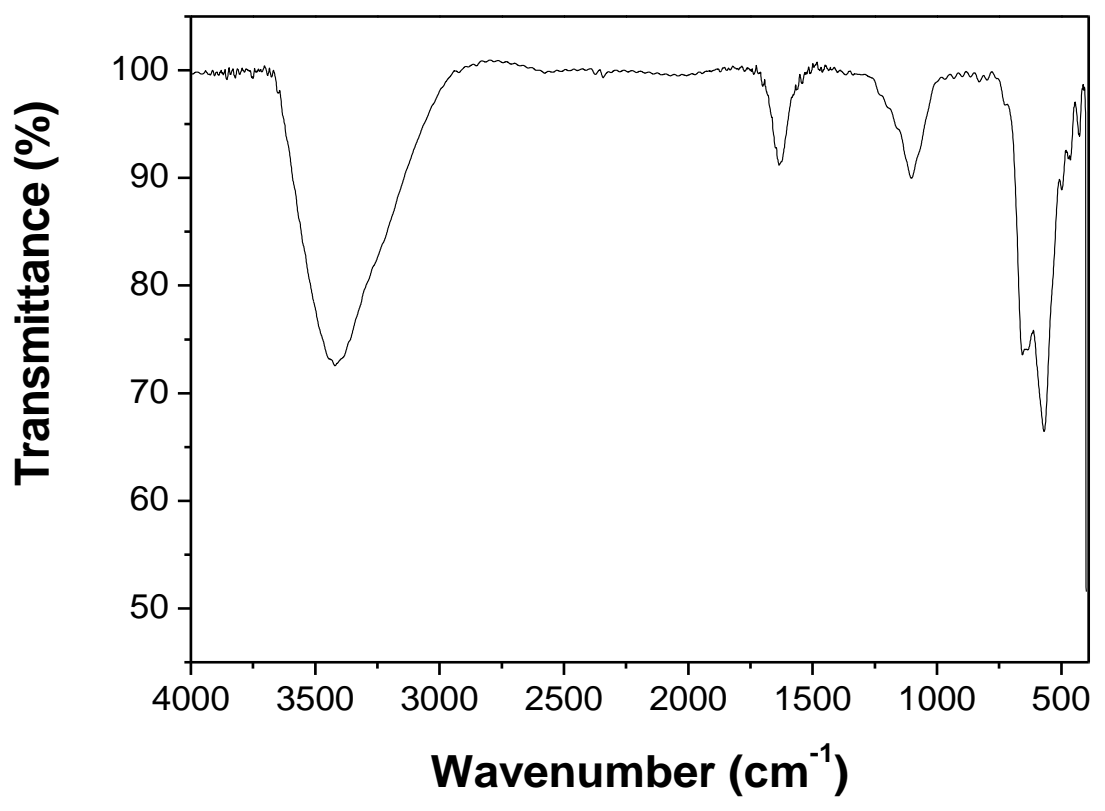

Figura 2 - Espectro de FTIR da $\mathrm{FeCO}_{2} \mathrm{O}_{4}$

\section{CONCLUSÃO}

A preparação do espinélio de cobaltita de ferro foi realizada com sucesso, proporcionando a obtenção do catalisador magnético $\mathrm{FeCo}_{2} \mathrm{O}_{4}$. Espera-se que o material desempenhe elevada eficiência na decomposição do poluente orgânico, possibilitando assim, uma inovação na área ambiental no que tange a síntese de materiais para remediação de contaminantes orgânicos em efluentes líquidos.

\section{AGRADECIMENTOS}

A Universidade Franciscana, pela oportunidade da bolsa de Iniciação Cientifica à acadêmica.

\section{REFERÊNCIAS}

BOCZKAJ, G.; FERNANDES, A. Wastewater treatment by means of advanced oxidation processes at basic $\mathrm{pH}$ conditions: A review. Chemical Engineering Journal, v. 320, p. 608-633, 2017.

BOKARE, A. D.; CHOI, W. Review of iron-free Fenton-like systems for activating $\mathrm{H}_{2} \mathrm{O}_{2}$ in advanced oxidation processes. Journal of hazardous materials, v. 275, p. 121-135. 2014.

DA SILVA, W. L.; LANSARIN, M. A.; MORO, C. C. Síntese, caracterização e atividade fotocatalítica de catalisadores nanoestruturados de $\mathrm{TiO}_{2}$ dopados com metais. Química Nova, v. 36, n. 3, p. 382-386, 2013. 
LIU, J.; NAN, Y.; CHANG, X.; LI, X.; FANG Y.; LIU, Y.; TANG, Y.; WANG, X.; LI, R.; $M A, J$. Hierarchical nitrogen-enriched porous carbon materials derived from Schiffbase networks supported $\mathrm{FeCo}_{2} \mathrm{O}_{4}$ nanoparticles for efficient water oxidation. International Journal of Hydrogen Energy, v. 42, p. 10802-10812, 2017.

MAEZONO, T.; TOKUMURA, M.; SEKINE, M.; KAWASE, Y. Hydroxyl radical concentration profile in photo-Fenton oxidation process: generation and consumption of hydroxyl radicals during the discoloration of azo-dye Orange II. Chemosphere, v. 82, p. 1422-1430, 2011.

OLIVEIRA, J. S.; BRONDANI, M.; MALLMANN, E.; JAHN, S.; FOLETTO, E.; SILVESTRI, S. Preparation of Highly Efficient $\mathrm{CoFe}_{2} \mathrm{O}_{4} / \mathrm{Zn}_{2} \mathrm{SnO}_{4}$ Composite Photocatalyst for the Degradation of Rhodamine B Dye from Aqueous Solution. Water air and soil pollution. , v. 229, p.1/386 - 9, 2018.

OLIVEIRA, J. S.; DRUMM, F. C.; MAZUTTI, M. A.; FOLETTO, E. L. ; JAHN, S. L. Preparação do sistema $\mathrm{Fe}_{2} \mathrm{O}_{3} / Z \mathrm{SM}-5$ para uso como catalisador na reação fotoFenton. Cerâmica, v. 62, p. 281-287, 2016.

OLIVEIRA, J. S.; HALMENSCHLAGER, F. C.; JAHN, S. L.,; FOLETTO, E. L. Síntese de $\mathrm{CoFe} 2 \mathrm{O} 4$ sobre os suportes $\mathrm{MgAl}_{2} \mathrm{O}_{4}$ e ZSM-5 para uso na degradação de poluente orgânico pelo processo foto-Fenton heterogêneo sob irradiação visível e solar. Materia-Rio de Janeiro, v. 24, p. 1-10, 2019.

VELÁSQUEZ, M.; SANTANDER, I.P.; CONTRERAS, D.R.; YAÑEZ, J.; ZAROR, C.; SALAZAR, R.A.; PEREZ-MOYA, M.; MANSILLA, H.D. Oxidative degradation of sulfathiazole by Fenton and photo-Fenton reactions. J. Environ. Sci. Health, Part A, v. 49, p. $661-670,2014$.

ZHAO, L.; YANG, D.; MA, L.; FENG, X.; DING, H. An efficient heterogeneous catalyst of $\mathrm{FeCO}_{2} \mathrm{O}_{4} / \mathrm{g}-\mathrm{C}_{3} \mathrm{~N}_{4}$ composite for catalytic peroxymonosulfate oxidation of organic pollutants under visible light. Colloids and Surfaces A, 2021 no prelo.

ZHOU, T.; WUA, X.; MAOA, J.; ZHANGA, Y.; LIMB, T.; Rapid degradation of sulfonamides in a novel heterogeneous sonophotochemical magnetite-catalyzed Fenton-like (US/UV/ $/ \mathrm{Fe}_{3} \mathrm{O}_{4} /$ oxalate) system.Applied CatalysisB:Environmental, v. 160-161, p. 325-334, 2014. 